\title{
Introduction to Enterprise System Integration: Issues and Answers
}

\author{
Marinos Themistocleous \\ University of Piraeus, Greece \\ mthemist@unipi.gr
}

\author{
Gail Corbitt \\ California State University, USA \\ gail_corbitt@yahoo.com
}

\author{
Pamela Schmidt \\ Washburn University, USA \\ pamela.schmidt@washburn.edu
}

Over the years, this mini-track has been a forum to disseminate knowledge on Enterprise Systems. This minitrack runs for the $19^{\text {th }}$ year in a row at HICSS and seeks to explore issues, both academic and organizational, surrounding the integration of Enterprise Systems. During the last decades, organizations have turned to the adoption of Enterprise Resource Planning (ERP) applications, Service Oriented Architecture (SOA) and cloudbased solutions to address their integration challenges. An increasing body of academic research has been devoted to this important and continually evolving area. The academic literature, and to a good extent, the professional literature, has been focused on using ERP systems to consolidate and refine the business processes within an organization. Increasingly, organizations are linking their systems with supply-chain partner's systems in efforts to streamline and automate the entire supply-chain. Such inter-organizational systems typify a newer challenge of process integration across corporate boundaries.

On the near-horizon, service providers are investigating use of Blockchain-based financial technologies to support inter-organizational interactions for both financial records and shared record-keeping, information integrity. Previously, Service Oriented Architecture has emerged to address these problems, with ERP vendors recognizing its importance and adopting SOA solutions and practices. Recently, ERP industry has turned to Cloud Computing, building on SOA, to reap benefits such as lowering of CAPEX for clients and the exploring new business models such as pay-as-you-go.

The major ERP vendors are currently attacking these issues, frequently with proprietary solutions. A more active involvement by the academic research community should allow for the establishment of a more open and standardized SOA/Cloud environment. Academics should have a role leading the use of these technologies.
In this edition, four papers have been accepted for presentation at the conference.

The first paper was written by Jeroen Wolters and U. Yeliz Eseryel and it is entitled "Identifying the Critical Success Factors for Low Customized ERP System Implementations in SMEs". The paper introduces a new level of analysis of critical success factors that was not previously considered in the normative literature. This deals with the level of ERP customization. The authors collect and analyze data from 216 SMEs and identify critical success factors for low customized ERP systems in SMEs and reveal few differences compared to the literature.

The second paper is entitled "Instantiations of an ERP Implementation Methodology: A Critical Realist Case Study" and was written by Daniela and Marius Mihailescu. The article adopts a qualitative research methodology to investigate the emergence of an ERP implementation methodology instantiations through the lens of a pragmatist social theory. The paper has both practical and theoretical contribution.

Gregor Friedrich-Baasner, Marcus Fischer and Axel Winkelmann are the authors of the third paper of our minitrack. The paper is entitled "Cloud Computing in SMEs: A Qualitative Approach to Identify and Evaluate Influential Factors", and it focuses on the identification of factors that influence the adoption of cloud computing services by Small and Medium Enterprises(SMEs). The paper is based on qualitative expert interviews and reveals that trust, privacy and security are the most significant influencing factors.

The last paper was written by Euripides Loukis and Niki Kyriakou, and it is entitled "Contractual and Relational Governance, ICT Skills and Organization Adaptations, and Cloud Computing Benefits". The paper studies the same area as the previous paper and it investigates factors that influence cloud computing adoption. The paper is based on data from 115 Greek companies and discloses interested findings and implications for practice and research. 